\title{
Asymptotic Locally Optimal Detector for Large-Scale Sensor Networks Under the Poisson Regime
}

\author{
Youngchul Sung, Lang Tong, Fellow, IEEE, and Ananthram Swami, Senior Member, IEEE
}

\begin{abstract}
We consider distributed detection with a large number of identical binary sensors deployed over a region where the phenomenon of interest (POI) has spatially varying signal strength. Each sensor makes a binary decision based on its own measurement, and the local decision of each sensor is sent to a fusion center using a random access protocol. The fusion center decides whether the event has occurred under a global size constraint in the Neyman-Pearson formulation. Assuming homogeneous Poisson distributed sensors, we show that the distribution of "alarmed" sensors satisfies the local asymptotic normality (LAN). We then derive an asymptotically locally most powerful (ALMP) detector optimized jointly over the fusion form and the local sensor threshold under the Poisson regime. We establish conditions on the spatial signal shape that ensure the existence of the ALMP detector. We show that the ALMP test statistic is a weighted sum of local decisions, the optimal weights being the shape of the spatial signal; the exact value of the signal strength is not required. We also derive the optimal threshold for each sensor. For the case of independent, identically distributed (iid) sensor observations, we show that the counting-based detector is also ALMP under the Poisson regime. The performance of the proposed detector is evaluated through analytic results and Monte Carlo simulations and compared with that of the counting-based detector. The effect of mismatched signal shapes is also investigated.
\end{abstract}

Index Terms-Asymptotically locally most powerful (ALMP), distributed detection, fusion rule, local asymptotic normality (LAN), Neyman-Pearson criterion, spatial Poisson process, spatially varying signal.

\section{INTRODUCTION}

\section{A. Detection in Large-Scale Sensor Field}

W E CONSIDER the detection of phenomenon in a geographical area using a large number of densely deployed microsensors. The sensors measure the phenomenon of interest (POI) and transmit their local data (the binary decision) via wireless channel to a central site for global processing. A specific implementation is the Sensor Network with Mobile Access

Manuscript received November 10, 2003; revised July 12, 2004. This work was supported in part by the Multidisciplinary University Research Initiative (MURI) under the Office of Naval Research under Contract N00014-00-1-0564. Prepared through collaborative participation in the Communications and Networks Consortium sponsored by the U.S. Army Research Laboratory under the Collaborative Technology Alliance Program, under Cooperative Agreement DAAD19-01-2-0011. The U.S. Government is authorized to reproduce and distribute reprints for Government purposes notwithstanding any copyright notation thereon. The associate editor coordinating the review of this manuscript and approving it for publication was Prof. Yucel Altunbasak.

Y. Sung and L. Tong are with the School of Electrical and Computer Engineering, Cornell University, Ithaca, NY 14853 USA (e-mail: ys87@ ece.cornell.edu; ltong@ece.cornell.edu).

A. Swami is with the Army Research Laboratory, Adelphi, MD 20783 USA (e-mail: a.swami@ieee.org).

Digital Object Identifier 10.1109/TSP.2005.847827

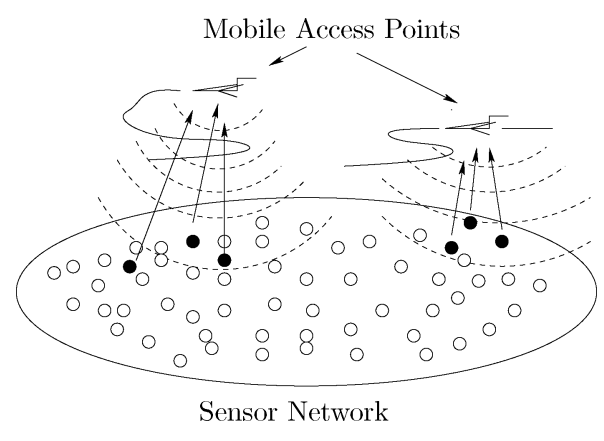

Fig. 1. Sensor network with mobile access points.

(SENMA) architecture [30], where a mobile access point or interrogator collects local decisions from sensors using random access schemes such as ALOHA; see Fig. 1. We assume that the number of sensors in the field is large, which makes it necessary that each sensor is inexpensive and has limited computation and communication capability.

Detection in a large-scale sensor network faces several challenges not encountered in the classical distributed detection problem. First, inexpensive sensors are not reliable; they have low duty cycles and severe energy constraints. The communication link between a sensor and the central unit is specially weak due to a variety of implementation difficulties such as synchronization, fading, and interference from other sensors. The probability that the local decision at a particular sensor can be successfully delivered to the central unit can be very low. Second, POI in a wide geographic area generates spatially varying signals, which makes the observation at each sensor location-dependent and not identically distributed. Furthermore, the strength of POI is unknown in many applications such as the detection of environmental hazards such as nuclear, biological, and chemical (NBC) activities. Third, the scale of the network makes it more practical to deploy sensors randomly without careful network layout. It is thus not possible to predict whether data from a particular sensor can be retrieved by the central processing unit, especially when random access protocols are used. Consequently, the decision rule for each sensor should be optimized before deployment without knowing its exact location and signal strength. In addition, because sensors may expire and the collection process is random, the optimal decision should not critically depend on the number of available sensors or on the collection process.

\section{B. Approach and Summary of Results}

For large-scale sensor networks it is natural to consider asymptotic techniques, and one expects that the central limit theorem will lead to a design under Gaussian statistics. For 
example, if the measurements at the sensors are conditionally independent and identically distributed (iid), it is well known that the global decision is made by counting the number of alarmed sensors collected from the sensor field, and the decision statistics will converge to a Gaussian random variable. When the measurements are not identically distributed across sensors or spatially varying, it is reasonable that the global detector should weight the decision of each sensor appropriately since sensors closer to the source (i.e., those with better SNR) produce more reliable decisions. For the example of detection of simple hypotheses, the weight is obtained by the local detection and false alarm probability at each sensor [16]. However, what should be the optimal weighting when the local detection or false alarm probability at each sensor is unknown? What are the factors affecting the weighting? Is there an assurance of asymptotic optimality?

Our approach is based on the local asymptotic normality (LAN) and asymptotic efficiency of detector in Le Cam sense. The LAN is a property of a statistical model itself not of detectors and simplifies the analysis and design of asymptotic procedures. The key idea is that if a sequence of statistical (parametric) models satisfies the LAN condition, then the sequence of statistical models is represented in the limit by Gaussian shift model where the analysis is much more tractable [1], [2]. (A brief summary of LAN-related results pertinent to our work is given in [31].) Our goal is to find decision rules for sensors and the central unit that are asymptotically efficient. Specifically, we find local and global decision rules that, for a given probability of false alarm (PFA) for the global decision, maximize the probability of detection as the number of sensors goes to infinity.

We model the POI over the region as a deterministic spatial signal with known shape but unknown signal strength. While the assumption of known signal shape is restrictive; the model of unknown signal strength is almost necessary in practice because it is unreasonable to assume that POI can be calibrated. From a theoretical point of view, not knowing the signal strength makes the detection problem more difficult and also more interesting in the asymptotic regime. For example, the direct use of error exponent to characterize performance as in [27] and [28] is no longer valid since the number of alternative hypothesis is uncountable. Indeed, if the signal strength is known, the error probability of any reasonable detector will always decay to zero as the number of collected sensor detections increases.

We assume that a Medium Access Control (MAC) protocol (such as ALOHA) is used to collect local decisions where each sensor has a probability $p_{m}$ to transmit its decision successfully to the central unit. In order to exploit spatial variation of the POI, it is necessary, as we shall assume, that the fusion center knows the location of each sensor through the use of a geolocation device at each sensor or other methods.

We assume that randomly deployed sensors form a homogeneous spatial Poisson process. For the model of independent additive noise at each sensor, the marking by the local decision of each sensor is equivalent to a location-dependent thinning of the initial Poisson process; the alarmed sensors form a nonhomogeneous Poisson process. The process of retrieving sensor decisions from the sensor field is another thinning of the alarmed sensors. The Poisson assumption allows us to combine the two thinning procedures at the physical and MAC layer and model the alarmed sensors at the central unit as a nonhomogeneous Poisson process with an intensity that is a function of the POI. Hence, the distributed detection problem is converted to detection based on the intensity of the observed alarmed sensors.

Applying the LAN of Le Cam, we derive 1) sufficient conditions on the spatial signal shape that guarantee the existence of asymptotically locally most power (ALMP) detector; 2) an asymptotic local upper bound (ALUB) on the power of any detector as a function of design parameters like sensor density, signal shape, MAC success probability, etc., and 3) an asymptotically locally jointly optimal rule over the fusion scheme and the single sensor threshold. For the special case that the power function of a single sensor is linear with respect to the signal strength, the proposed detector is also asymptotically uniformly most powerful.

Our numerical results are designed to answer a number of practical questions. Since the detector is based on asymptotic techniques, one questions what the size of network is for which the asymptotic analysis is accurate. The simulations show that the performance of a network of size 1000 matches well with the theoretic prediction. We will see that the proposed ALMP detector offers a significant gain over simplistic counting schemes. Since we assume the knowledge of signal waveform in the detector design, we also consider the case of waveform mismatch in our simulations. The sensitivity of the mismatch, of course, depends on the specific shape of the signal waveform. For the class of symmetric exponentially decaying waveforms, we find that a simple step function approximation offers graceful degradation. Further, we find that the performance is robust to perturbations in the assumed locations of the sensors.

\section{Related Work}

Distributed detection using multiple sensors and optimal fusion rules has been extensively investigated; see [12]-[14]. Many authors have derived optimal local detector and fusion rules under various scenarios based on different sets of assumptions, e.g., [15], [17], and [18]. For the fusion scheme, Chair and Varshney [16] showed that the optimal fusion rule is a likelihood ratio test on the decisions from the sensors and becomes a threshold detector on the weighted sum of binary sensor decisions; the weight is obtained using the local detection and PFA at each sensor under each hypothesis. However, the optimal criteria are obtained under the assumption that the hypotheses of the underlying phenomenon are simple, i.e., discrete and finite. These approaches require the knowledge of the detection probability as well as the PFA at each sensor under each possible hypothesis; however, in the problem considered here we do not have such information.

The detection of an unknown signal or a signal with unknown amplitude has been considered by several authors under the composite hypothesis formulation. The locally optimal detector for centralized schemes is known [20], [21]. Poor and Thomas considered the locally optimal detector for stochastic signals and compared the detectors using the asymptotic relative efficiency (ARE) of the centralized detection scheme [22]. For the 
distributed or decentralized case, Aalo and Viswanathan considered the detection of an unknown signal via multilevel quantization and simple fusion rules [24]. However, no optimality for the fusion rule was considered. The works of Fedele et al. [25] and Srinivasan [26] are perhaps the closest to our approach. In both cases, the authors considered a distributed scheme where multiple peripheral detectors or sensors are combined at a fusion center, and the number of observations per each sensor goes to infinity. These assumptions are reasonable for the classical radar problem. For large-scale microsensor networks, however, it is reasonable to assume that each sensor has only a few chances for observations and transmissions, due to limited battery power, and to consider the asymptotic case where the number of sensors goes to infinity, but with a limited number of observations per sensor as in this paper. Srinivasan derived the optimal local rule and fusion rule based on Bayes rule and summation over all realizations of sensor decisions [26]. However, it is difficult to extend this approach to yield explicit fusion rules for the scenario considered in this paper.

The asymptotic case where the number of sensors goes to infinity was also considered by several authors from different perspectives. For example, the error exponent was used as the asymptotic performance measure to show the optimality of identical sensors when the observations are iid [27], [28]. In [29], the authors considered the optimality of identical binary sensors for the capacity limited reachback channel.

\section{Notation}

The statistical experiment or model $(\Omega, \mathcal{X}, \mathcal{P})$ is described as follows. An event $X \in \mathcal{X}$ is observed such that the probability distribution of $X$ is from a parametric family of probability measures $\mathcal{P}=\left\{P_{\theta}, \theta \in \Theta\right\}$, all defined on the same measurable space $(\Omega, \mathcal{X})$; the true parameter $\theta$ is unknown. The statistical experiment or model is simply denoted by $\left\{P_{\theta}, \theta \in \Theta\right\}$. The sequence of statistical models is denoted by $\left(\Omega^{(n)}, \mathcal{X}^{(n)}, \mathcal{P}^{(n)}\right)$, where $\mathcal{P}^{(n)}=\left\{P_{\theta}^{(n)}, \theta \in \Theta\right\}$. Notice that the parameter space $\Theta$ does not change with the sequence index in our formulation, and the superscript $(n)$ does not denote the product space or measure in general. It can be an arbitrary sequence of measurable spaces and probability measures. For the product distribution of $n$ iid $P_{\theta}$, we use the notation $P_{\theta}^{\otimes n}$. For a sequence of random vectors $\mathbf{x}_{n}$ defined on $\Omega^{(n)}, \mathbb{E}_{n, \theta} \mathbf{x}_{n}$ is the statistical expectation of $\mathbf{x}_{n}$ under probability distribution $P_{\theta}^{(n)}$. The notation $\mathbf{x} \sim \mathcal{N}(\boldsymbol{\mu}, \boldsymbol{\Sigma})$ means that $\mathbf{x}$ is Gaussian with mean $\boldsymbol{\mu}$ and covariance $\Sigma$. The set of real numbers is denoted by $\mathbb{R}$. Vectors and matrices are written in boldface. Operation $(\cdot)^{T}$ indicates the matrix transpose.

\section{SYSTEM MODEL}

We consider a large-scale sensor network with identical binary sensors deployed over a wide area; we want to decide whether the POI has occurred in the area. We assume that each sensor makes a decision based on its own observation and that the local decisions are collected through a MAC at a central

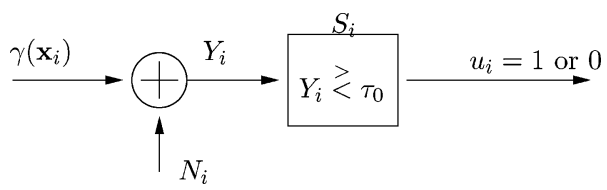

Fig. 2. Sensor observation model at location $\mathbf{x}_{i}$.

unit or fusion center where the global decision is made under a level(PFA) constraint. The POI is spatially varying with a known $^{1}$ shape function and an unknown magnitude. For example, in the case of NBC activity the signal strength is expected to be largest at the origin of the phenomenon and to decay away from the origin. We assume that the spatial signal is deterministic and denote the signal strength by

$$
\gamma(\mathbf{x})=\theta s(\mathbf{x})
$$

where $\mathbf{x}$ denotes the location, $\theta \in \Theta \triangleq[0, \infty)$ is an unknown amplitude, and $s(\mathbf{x})$ is a known function which incorporates the information about the spatial variation of the underlying phenomenon.

\section{A. Single Sensor}

We assume that sensors make their local decisions independently without collaborating with other sensors. Since the exact value of the signal strength is unknown, we design each sensor to decide between the following (composite) hypotheses

$$
\begin{array}{lll}
H_{0}: & \gamma(\mathbf{x})=0 & \text { (null hypothesis) } \\
H_{1}: & \gamma(\mathbf{x})>0 & \text { (alternative hypothesis) }
\end{array}
$$

with local size constraint of $\alpha_{0}$. Using the amplitude parameter $\theta$, the hypotheses (2) is equivalently expressed by

$$
\begin{array}{ll}
H_{0}: & \theta=0 \\
H_{1}: & \theta>0 .
\end{array}
$$

The local decision of sensor $S_{i}$ located at $\mathbf{x}_{i}$ is denoted by

$$
u_{i}= \begin{cases}1, & \text { if } H_{0} \text { is rejected } \\ 0, & \text { otherwise. }\end{cases}
$$

We consider the additive noise model for sensor measurement shown in Fig. 2, where the sensor observation $Y_{i}$ is given by

$$
Y_{i}=\gamma\left(\mathbf{x}_{i}\right)+N_{i}
$$

where $N_{i}$ is the sensor measurement noise, assumed to be independent across sensors, with distribution $p_{N}(\nu)$. Here, we assume that the distribution $p_{N}(\nu)$ admits a local UMP detector at the sensor level for hypotheses (2) as

$$
u_{i}= \begin{cases}1, & Y_{i} \geq \tau_{0} \\ 0, & Y_{i}<\tau_{0}\end{cases}
$$

${ }^{1}$ We need not assume that the shape function $s(\mathbf{x})$ is known a priori before sensor deployment or at the data retrieval period. See Section III-D for the estimation of $s(\mathbf{x})$ from collected binary sensor decisions and sensor locations. This includes the case where the shape function is parameterized, with unknown parameters that must be estimated. 


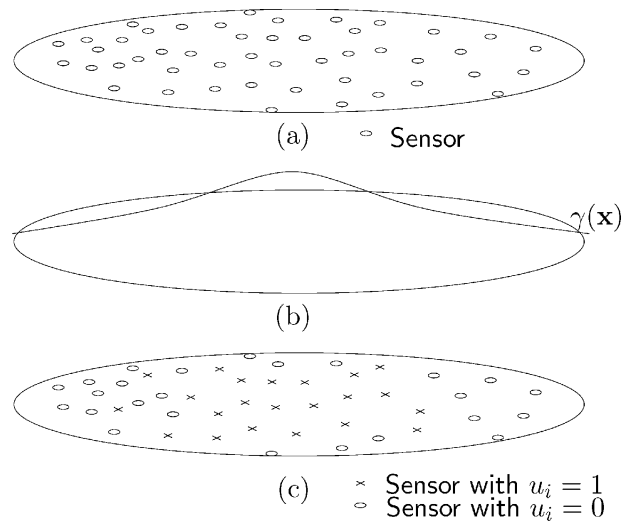

Fig. 3. (a) Initial sensor deployment over space. (b) Signal strength of underlying phenomenon. (c) Local decisions of sensors.

where $\tau_{0}$ is chosen to satisfy the size $\alpha_{0}$ for a single sensor decision. Such $p_{N}(\nu)$ includes continuous symmetric distribution around zero that decreases monotonically as $\nu$ increases from zero. For the example of Gaussian noise model $N_{i} \sim \mathcal{N}\left(0, \sigma_{0}^{2}\right)$, we have the threshold $\tau_{0}=\sigma_{0} Q^{-1}\left(\alpha_{0}\right)$, where $Q(x)$ denotes the tail probability $Q(x)=(1 / \sqrt{2 \pi}) \int_{x}^{\infty} e^{-(1 / 2) t^{2}} d t$.

We define $p(\mathbf{x})$ as the probability that a sensor located at $\mathbf{x}$ rejects $H_{0}$. That is,

$$
p\left(\mathbf{x}_{i}\right)=\operatorname{Pr}\left\{u_{i}=1\right\} .
$$

Note that this probability is not conditioned on hypothesis $H_{0}$ or $H_{1}$. The probability $p(\mathbf{x})$ is a function of the local threshold and the signal strength at $\mathbf{x}$ and is given by

$$
p(\mathbf{x})=\beta_{\tau_{0}}(\gamma(\mathbf{x})) .
$$

Dependence on the pdf of the observation noise is through the threshold $\tau_{o}$. For the additive Gaussian observation model, $\beta_{\tau_{0}}(\gamma(\mathbf{x}))$ is expressed as $Q\left(\left(\tau_{0}-\gamma(\mathbf{x})\right) / \sigma_{0}\right)$.

\section{B. Parametric Poisson Model}

Consider that a large number of identical sensors described in Section II-A are deployed randomly and uniformly over a region $A$, as shown in Fig. 3(a). We assume that the initial distribution of sensors over the region is a homogeneous spatial Poisson process with local intensity $\lambda_{h}$. This is a reasonable model when the random location of each sensor is uniformly distributed over $A$. Each sensor makes a local decision about the underlying phenomenon. Specifically, sensor $S_{i}$ located at $\mathbf{x}_{i}$ makes a binary decision $u_{i}$ based on its observation, encodes its decision, and then sends its packet over a MAC to the central unit.

Since we assume that each sensor makes the decision by itself and the sensor noise is independent, the local decision $u_{i}$ is independent conditioned on the signal strength $\gamma(\mathbf{x})$. By the Poisson assumption on the initial sensor locations, the marking by the local decision of each sensor is equivalent to a location-dependent thinning of the original sensor distribution with thinning probability $p(\mathbf{x})$. Hence, the distribution of the alarmed sensors, i.e., sensors with $u_{i}=1$, forms a nonhomogeneous spatial Poisson process.

During the data-retrieval period the local decisions of the sensors are collected through wireless channel. Sensor data can be lost during the transmission due to fading as well as collisions. We model this probabilistic loss as another thinning of the Poisson process of alarmed sensors (PPAS). We assume that all the sensors have the same probability of successful transmis$\operatorname{sion}^{2}$ and denote it by $p_{m}$. Then, the second thinning is uniform over the region with probability $p_{m}$. The distribution of alarmed sensors at the fusion center or final data collector is a nonhomogeneous spatial Poisson process, and its local intensity is given by

$$
\begin{aligned}
\lambda(\mathbf{x}) & =\lambda_{h} p_{m} p(\mathbf{x})=\lambda_{h} p_{m} \beta_{\tau_{0}}(\theta s(\mathbf{x})) \\
& =\lambda_{h} p_{m}\left[\beta_{\tau_{0}}(0)+\beta_{\tau_{0}}^{\prime}(0) \theta s(\mathbf{x})+o(\theta)\right]
\end{aligned}
$$

where $\beta_{\tau_{0}}^{\prime}(\gamma(\mathbf{x}))=(\partial / \partial \gamma(\mathbf{x})) \beta_{\tau_{0}}(\gamma(\mathbf{x}))$. When the function $\beta_{\tau_{0}}(\cdot)$ is linear, or $\theta$ is in a small neighborhood of $\theta=0$ (i.e., the signal is weak), the Poisson process of alarmed sensors is described by a nonhomogeneous intensity model parameterized by amplitude $\theta$ given by

$$
\lambda(\theta, \mathbf{x})=\theta f(\mathbf{x})+\lambda_{0}, \quad \theta \in \Theta
$$

where

$$
f(\mathbf{x})=\lambda_{h} p_{m} \beta_{\tau_{0}}^{\prime}(0) s(\mathbf{x}), \quad \lambda_{0}=\lambda_{h} p_{m} \beta_{\tau_{0}}(0)
$$

for a given $\tau_{0}$. Note that the intensity variation $f(\mathbf{x})$ of alarmed sensors is a scaled version of the spatial signal shape $s(\mathbf{x})$.

Since the initial sensor distribution is assumed to be Poisson, we are able to convert the original detection problem to one of detecting an inhomogeneous Poisson process whose intensity function depends upon the spatial signal at the alarmed sensors. (This is what we mean by "Poisson regime" in this paper.) As we will show later, the asymptotic detector requires the knowledge of the signal $s(\mathbf{x})$ and of the location of the reporting sensors; however, the asymptotic detector will be shown to be surprisingly robust with respect to the shape function $s(\mathbf{x})$ as well as its "origin."

\section{Detection of Spatially VARYing Signal}

In the previous section, we assumed that the initial sensor distribution is Poisson and showed that the original detection problem using identical binary sensors is converted to the problem (10), (11), and (3) of detecting Poisson processes with different intensities.

Under asymptotic local optimality we focus on the detection of the alternative which converges to the null hypothesis $\theta=0$, where the distributions of the null and alternative hypotheses are not entirely asymptotically separated (EAS). The existence of an asymptotic locally optimal detector (ALOD) requires some conditions on the underlying statistical model. Le Cam's theory provides an analytic framework for such detection problems and gives an asymptotic optimal criterion. When a statistical model satisfies the LAN condition [1], [2], [4], we can construct an asymptotic local upper bound on the power of any sequence of detectors with a given asymptotic size, and we can construct a sequence of detectors that achieves this bound.

${ }^{2}$ The equal probability assumption for successful transmission may be restrictive. However, this gives a reasonable approximation when the data collector has control over sensor transmissions. 
In Appendix I, we construct a sequence of statistical models for PPAS and establish its LAN property. Based on this, we obtain an ALUB on the power of any detector with a given size, and we derive the ALMP detector for the model (10), (11), and (3) under the Poisson regime, as the number of sensors goes to infinity for a fixed area.

\section{A. ALMP Detector Under the Poisson Regime}

In Appendix I, we show that the Poisson process of the alarmed sensors, with increasing sensor density, satisfies the LAN condition for a general family of signal shapes. The construction of an ALMP detector is then straightforward. An ALMP detector is given by the score test using the central sequence [4], [31].

Theorem 1: Let conditions C.1)-C.3) be satisfied.

C.1) $f(\mathbf{x}) \geq 0$, and $\mathbf{x} \in A$;

C.2) $\sup _{\mathbf{x} \in A} f(\mathbf{x})<\infty$;

C.3) $\int_{A} f(\mathbf{x}) d \mathbf{x}>0$;

where $f(\mathbf{x})$ is defined in (11). Then, an asymptotic upper bound on the power of any sequence of detectors $\phi_{n}$ with size $\alpha$, i.e., $\limsup _{n \rightarrow \infty} \mathbb{E}_{n, 0} \phi_{n} \leq \alpha$, is given by

$$
\limsup _{n \rightarrow \infty} \sup _{0<r_{n}(0)^{-1}}\left[\mathbb{E}_{n \leq M}, \theta \phi_{n}-Q\left(Q^{-1}(\alpha)-r_{n}^{-1}(0) \theta\right)\right] \leq 0
$$

for any $M>0$, where

$$
r_{n}(0)=\left(n \lambda_{h 0} p_{m} \frac{\left(\beta_{\tau_{0}}^{\prime}(0)\right)^{2}}{\beta_{\tau_{0}}(0)} \int_{A} s^{2}(\mathbf{x}) d \mathbf{x}\right)^{-1 / 2} .
$$

Furthermore, the following sequence of (nonrandomized) detectors is ALMP with size $\alpha$ for (10), (11), and (3).

$$
\phi_{n, o p t}= \begin{cases}\text { Decide } H_{0}, & \text { if } \Delta_{n, 0} \leq Q^{-1}(\alpha) \\ \text { Decide } H_{1}, & \text { if } \Delta_{n, 0}>Q^{-1}(\alpha)\end{cases}
$$

where the central sequence $\Delta_{n, 0}$ is given by

$$
\begin{aligned}
\Delta_{n, 0}=n^{-1 / 2} \lambda_{0}^{-1 / 2} & \left(\int_{A} s^{2}(\mathbf{x}) d \mathbf{x}\right)^{-1 / 2} \\
& \times\left(\sum_{\mathbf{x}_{i} \in A} s\left(\mathbf{x}_{i}\right)-n \lambda_{0} \int_{A} s(\mathbf{x}) d \mathbf{x}\right)
\end{aligned}
$$

and $\mathbf{x}_{i}$ 's are the (random) locations of alarmed sensors in the area $A .^{3}$.

Proof: See Appendix II.

The conditions in Theorem 1 are mild and are discussed in Appendix I. Equations (12) an (13) reveal how various factors such as sensor density $n \lambda_{h 0}$, the probability of packet loss $p_{m}$, the spatial signal shape $s(\mathbf{x})$, and the single sensor threshold $\tau_{o}$, affect the asymptotic global power. Note that as expected, the power of the detector increases monotonically with sensor density, signal strength, and MAC transmission success rate. We observe that if the signal strength is halved, sensor density must be quadrupled in order to maintain the asymptotic performance. This is consistent with the notion of fusing independent signal

\footnotetext{
${ }^{3} n$ is not the number of sensors. It is the index for the statistical experiment described Appendix I-B. The average number of sensors in $A$ is given by $n \lambda_{h 0}$.
}

decisions. Since the ALMP test statistic (the central sequence $\left.\Delta_{n, 0}\right)$ has a limit distribution $\mathcal{N}(0,1)$ under the null distribution $P_{0}^{(n)}$ by the LAN condition, it is easy to see that the detector (14) has an asymptotic size of $\alpha$. Notice that the ALMP test statistic consists of a weighted sum of binary sensor decisions, where the optimal weight is $s(\mathbf{x})$, which is the shape of underlying spatial signal $\gamma(\mathbf{x})$. Thus, the confidence in each sensor decision is proportional to the strength of the signal (the SNR) at the sensor location. This can be considered as matched filtering in the spatial domain, although it is different from the conventional matched filtering in that the received signal is a collection of random points with an intensity function determined by the input signal, whereas the received signal is simply the distorted version of the input signal for the conventional case. A related problem and approach is in [11], where the author considered a binary on-off detection problem in optical transmissions. The author assumed that the photon generation epochs were Poisson points and showed that the optimal weight is the intensity of input light under a Bayesian formulation of two simple hypotheses. However, the exact knowledge of the intensity of light was required rather than just the relative shape. In our proposed method, the optimal test can be implemented without obtaining the exact value of $\gamma(\mathbf{x})$, since the optimal weight requires only the local intensity variation $s(\mathbf{x})$ of alarmed sensors, and any scaling of $s(\mathbf{x})$ is irrelevant in forming the statistic (15).

An intuitive interpretation is given by a step function given by

$$
s(\mathbf{x})= \begin{cases}1, & \mathbf{x} \in A_{1}(\in A) \\ 0, & \mathbf{x} \in A \backslash A_{1}\end{cases}
$$

where $A \backslash A_{1}$ is the difference set. In this case, the local decisions from only the sensors located within the region $A_{1}$, where the phenomenon would occur, are counted discarding the false alarms from the regions of no event. For more complicated signal shapes such as (25), the local decisions from sensors are weighted according to the relative strength of the underlying signal.

\section{B. Optimization of Threshold for a Single Sensor}

In Section III-A, we derived an ALMP detector. The optimal local threshold for a single sensor described in Section II-A is now obtained through the asymptotic (local) upper bound (12) and (13). Since the bound is a function of the local threshold and achieved by the proposed ALMP detector, the optimal threshold for a single sensor is the one that maximizes the asymptotic upper bound. In Section IV, we show that the asymptotic bound can be achieved with a reasonable number of sensors.

Theorem 2: Suppose that the power function $\beta_{\tau_{0}}(\gamma(\mathbf{x}))$ for a single sensor is continuous and piecewise differentiable in the variable $z=\gamma(\mathbf{x})$. Under the Poisson regime, the following threshold for a single sensor maximizes the global power for a fixed and sufficiently large number of sensors in the region

$$
\tau_{o p t}=\underset{\tau_{0}}{\arg \max } \frac{\beta_{\tau_{0}}^{\prime}(0)^{2}}{\beta_{\tau_{0}}(0)}
$$

where $\beta_{\tau_{0}}^{\prime}(\gamma(\mathbf{x}))=(\partial / \partial \gamma(\mathbf{x})) \beta_{\tau_{0}}(\gamma(\mathbf{x}))$

Proof: See Appendix II. 
For the example of Gaussian noise model, we have $\beta_{\tau_{0}}(\gamma(\mathbf{x}))=Q\left(\left(\tau_{0}-\gamma(\mathbf{x})\right) / \sigma_{0}\right), \beta_{\tau_{0}}(0)=Q\left(\tau_{0} / \sigma_{0}\right)$, and $\beta_{\tau_{0}}^{\prime}(0)=\left(1 / \sqrt{2 \pi} \sigma_{0}\right) \exp \left(-(1 / 2)\left(\tau_{0} / \sigma_{0}\right)^{2}\right)$. The optimal local size is $\alpha_{0}=0.27$, and the corresponding local threshold $\tau_{o}=0.612 \sigma_{o}$ (we verify this via simulations in Section IV). Notice that under the assumption of binary decisions and Poisson distributed sensors, the individual sensors need not be very good; a design with a PFA of 0.27 is optimal! We also note that for the AWGN model, $\beta_{\tau_{0}}^{\prime}(0)^{2} / \beta_{\tau_{0}}(0)$ is fairly flat around its maximum so that it is not critical to use the optimal $\tau_{o}$ and $\alpha_{o}$ values. The threshold for the AWGN case coincides with the one obtained for nonparametric detection of symmetric distribution using dead-zone limiter with iid observations [23]. This implies that we should design the single sensor assuming the signal shape is uniform over the area if the information of the signal shape is not available before sensor deployment and identical sensors are to be deployed over the field. (This is the case that we consider in Section II-B.) In this case, the local sensors need not know the signal shape; further, the optimal local threshold does not depend on the signal shape $s(\mathbf{x})$. Thus, a deployed set of sensor nodes can be used to detect different signatures, and only the fusion center needs to know the signature.

Notice that the optimal fusion rule (15) and the local threshold (17) do not depend on the parameter $\theta$. Hence, the optimal rule is actually an asymptotically uniformly most powerful detector when the model (10) is true for all $\theta \in \Theta$, for example, when the power function for a single sensor is linear in $\theta$. However, in general, our conversion to the Poisson regime is valid in the local neighborhood of $\theta=0$ for a typical power function $\beta_{\tau_{0}}(\cdot)$.

\section{Independent and Identically Distributed Observations}

If the signal is constant,

$$
s(\mathbf{x}) \equiv 1, \quad \mathbf{x} \in A
$$

then the sensor observations, for the model described in Section II, become iid. The optimality of the counting-based detector is given by the following corollary of Theorem 1 .

Corollary 1: For iid sensor observations over $A$, the counting-based detector is ALMP with size $\alpha$ under the Poisson regime.

Proof: In this case, the central sequence is given by [see (15)]

$$
\Delta_{n, 0}=\left(n \lambda_{0}|A|\right)^{-1 / 2}\left(N^{(n)}(A)-n \lambda_{0}|A|\right)
$$

where $N^{(n)}(A)$ is the number of alarmed sensors in space $A$, and $|A|$ is the area of $A$.

Note that $N^{(n)}(A)$ is a Poisson random variable with mean $n \lambda_{0}|A|$ under the null hypothesis. Since the mean and variance are equal for Poisson random variables, $\Delta_{n, 0}$ is centered and normalized to have variance one. The Gaussian limit distribution of $\Delta_{n, 0}$ under the null hypothesis is explained as follows. Suppose that we partition $A$ into $n$ subregions with an equal area for the $n$th experiment. Under the null hypothesis, we have $\lambda^{(n)}(0, \mathbf{x})=n \lambda_{0}$ from (34) in Appendix I. Therefore, the number of alarmed sensors in each subregion is a Poisson random variable with mean $\lambda_{0}|A|$ regardless of $n$ and is iid over subregions under the Poisson assumption. Since $N^{(n)}(A)$ is the sum of number of points in each subregion, it is a sum of $n$ iid random variables, and $\Delta_{n, 0}$ converges in distribution to $\mathcal{N}(0,1)$ as $n$ goes to infinity by the classical central limit theorem (CLT).

A different counting-based detector can be constructed for the iid case based on the Binomial distribution [19]. Let the number of sensors in $A$ be $K$. Under $H_{0}, \gamma(\mathbf{x})=0$ for all $\mathbf{x}$ and

$$
u_{i} \stackrel{i i d}{\sim} B\left(1, p_{0}\right), p_{0}=\operatorname{Pr}\left\{u_{i}=1 \mid \gamma(\mathbf{x})=0\right\}=\beta_{\tau_{0}}(0) .
$$

Recall that the $u_{i}$ are the binary sensor decisions. Using the CLT, the asymptotic distribution of $\sum_{i=1}^{K} u_{i}$ is given by

$$
\frac{1}{\sqrt{K p_{0}\left(1-p_{0}\right)}}\left(\sum_{i=1}^{K} u_{i}-K p_{0}\right) \stackrel{D}{\Rightarrow} \mathcal{N}(0,1)
$$

as the number of sensors $K$ goes to infinity. Hence, the detector is given by

$$
\text { Reject } H_{0} \text { if } \sum_{i=1}^{K} u_{i}>K p_{0}+Q^{-1}(\alpha) \sqrt{K p_{0}\left(1-p_{0}\right)} \text {. }
$$

The distributions for the number of alarmed sensors in (19) and (21) have different variances for the same mean (i.e., $n \lambda_{0}|A|=$ $K p_{0}$ ) under two different models. It is well known that the binomial distribution converges to Poisson distribution with mean $\Lambda$ when $K$ goes to infinity with constraint $K p_{0}=\Lambda$. Therefore, when $p_{0}$ is small, the two distributions are almost equivalent.

\section{Discussion}

The construction of the ALMP test statistic $\Delta_{n, 0}$ in (15) requires the knowledge of several parameters such as the null intensity $\lambda_{0}$, the sensor locations, and the shape of the underlying spatial signal, but the null intensity can be obtained from the known or controllable parameters such as the density of the initial sensor distribution, the local PFA $\alpha_{0}$, and the probability of successful transmission through the MAC $p_{m}$. Here, we briefly discuss the estimation of $s(\mathbf{x})$.

As shown in Theorem 1, the ALMP weight is the spatial signal shape $s(\mathbf{x})$ under the Poisson regime. We consider an estimation method based on the collected sensor data. One simple way is to utilize the Poisson assumption itself. Equation (10) reveals that $f(\mathbf{x})$ is the local intensity variation of alarmed sensor distribution over space. Hence, the weight can be estimated from the alarmed sensors and their location directly. For example, we can use a nonparametric intensity model

$$
f(\mathbf{x})=\sum_{j} f_{j} I_{A_{j}}, \bigcup_{j} A_{j}=A, A_{i} \cap A_{j}=\phi .
$$

Assuming that $\lambda_{0}$ is known, the maximum likelihood estimator of $\theta f(\mathbf{x})$ for the model (23) is given by

$$
\widehat{\theta f(\mathbf{x})}=\sum_{j} \frac{N\left(A_{j}\right)}{\left|A_{j}\right|} I_{A_{j}}-\lambda_{0} .
$$

Since any scaling of $s(\mathbf{x})$ does not matter for obtaining $\Delta_{n, 0}$, $\theta \widehat{f(\mathbf{x}})$ can be used as an estimate $\hat{s}(\mathbf{x})$ for the optimal weight function. For this estimation method, however, several independent measurements by sensors are required, and this method is 
useful only when $\theta$ is fairly large. We are currently investigating more efficient methods to estimate the signal variation based only on the binary sensor decisions and sensor locations. The performance degradation due to an incorrectly assumed signal shape is investigated in Section IV-B.

\section{NUMERICAL RESUlts}

In this section, we present some simulation results. We used the receiver operating characteristics (ROC) as the performance criterion. The power of the proposed ALMP detector was evaluated via the analytic results in (12), (13) and by Monte Carlo simulations. The PFA was also estimated to check the validity of detector design in the Neyman-Pearson context. The power of the proposed detector (Theorem 1) was compared with that of the counting-based detector (22), which also has an asymptotic size of $\alpha$ but does not exploit the spatial information. Performance degradation due to mismatched signal shapes was also investigated.

\section{A. Setup}

We considered a two-dimensional (2-D) space $A$ that is circular with unit radius. The spatial signal shape was the symmetric exponential given by

$$
s(x, y)=e^{-\eta r}, r=\sqrt{x^{2}+y^{2}}
$$

with a decay rate $\eta>0$. The average number of sensors in $A$ was chosen to be 1000 . For the local sensor function, we used the additive Gaussian noise model and the UMP detector with several local sizes (see Section II-A).

For the simulation of power and PFA, 10000 Monte Carlo runs were executed. For each run, the following procedures were performed. The locations of the sensors were randomly generated according to a homogeneous Poisson process with the given mean intensity. For each sensor, we implemented the detector (6) for the AWGN model with noise variance $\sigma_{o}^{2}=1$. Global decision statistics are given by (15) for the ALMP detector and by (19) for the counting-based detector. The global thresholds for both detectors were determined via the Gaussian limit distribution. Throughout the simulations, the probability of successful data collection from each sensor was set to $p_{m}=0.9$. The initial homogeneous density $\lambda_{h}$ and the local PFA were assumed to be known and the true values were used for the simulation.

\section{B. Receiver Operating Characteristics}

Fig. 4 shows the analytic upper bound on the power versus the number of sensors for global size 0.1. It also depicts the expected behavior that if the signal strength $\theta$ decreases, we need more sensors to achieve the same performance. Fig. 5 shows the analytic bound and simulated power with respect to the PFA. For the simulation curve, the actual PFA was used rather than the designed size. As shown in the figure, the power of ALMP detector achieves the asymptotic upper bound with a network size of 1000 . Notice that the simulated power is slightly larger than the bound. This is because the Gaussian power function $\beta_{\tau_{0}}(\cdot)$ is convex in the range $\alpha_{0} \leq 0.5$ and larger than the linear

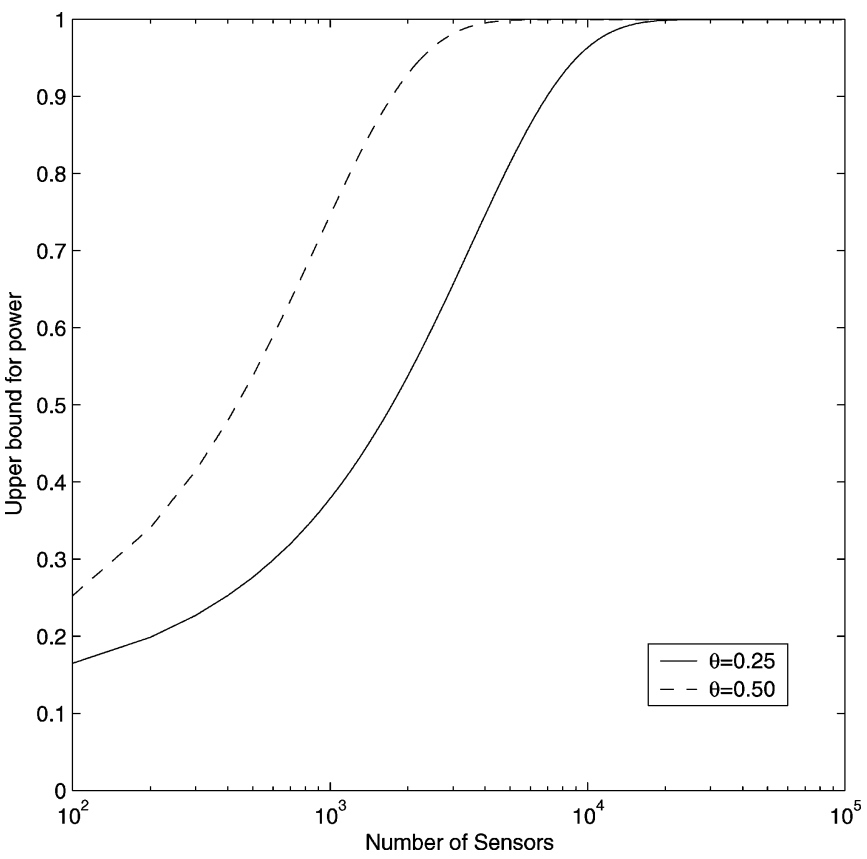

Fig. 4. Upper bound on power versus average number of sensors $(\eta=3)$.

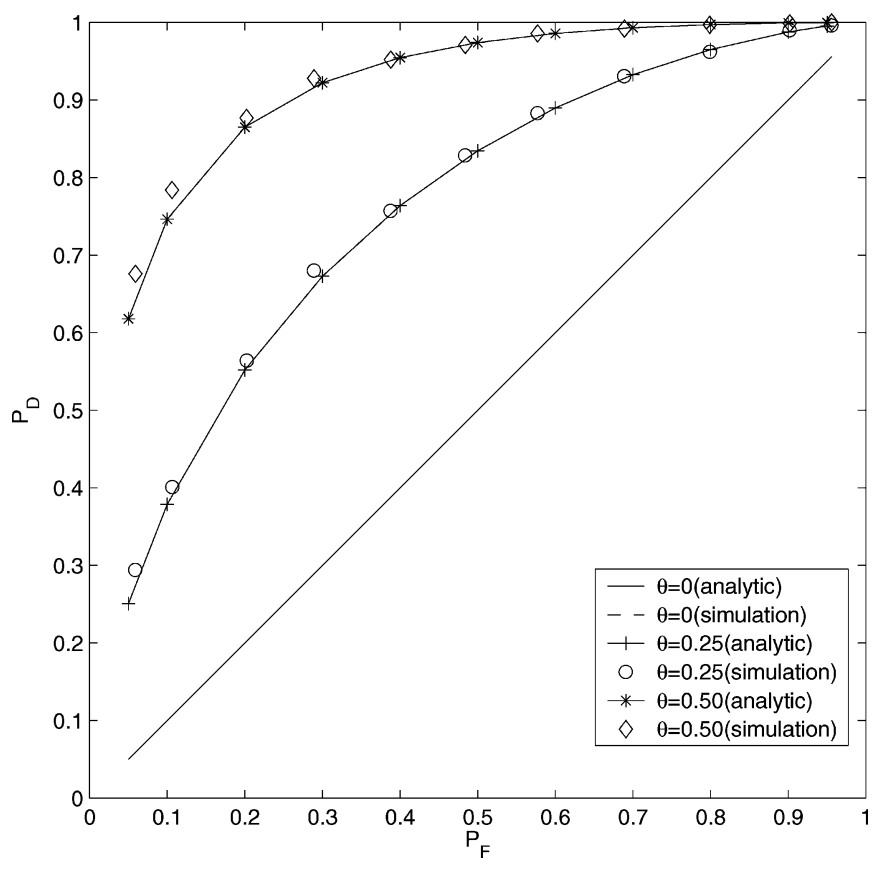

Fig. 5. ROC-Analytic and simulation curves $(\eta=3)$.

approximation at $\theta=0$. However, the figure shows that the difference is negligible and that the linear modeling (10) is valid in this range of $\theta$.

One important feature of the proposed detector is that the test statistic $\Delta_{n, 0}$ has a Gaussian limit distribution and the global threshold is based on it. Fig. 6 shows the actual PFA obtained by simulation versus the designed size for the additive Gaussian noise model with local size 0.1 and verifies the convergence in distribution of the test statistic with a network size of 1000 sensors. As shown, the actual PFAs closely follow the design size. Notice that the actual PFA of the ALMP detectors for decay rate $\eta=6$ deviates more than that for the case of $\eta=3$, whereas 


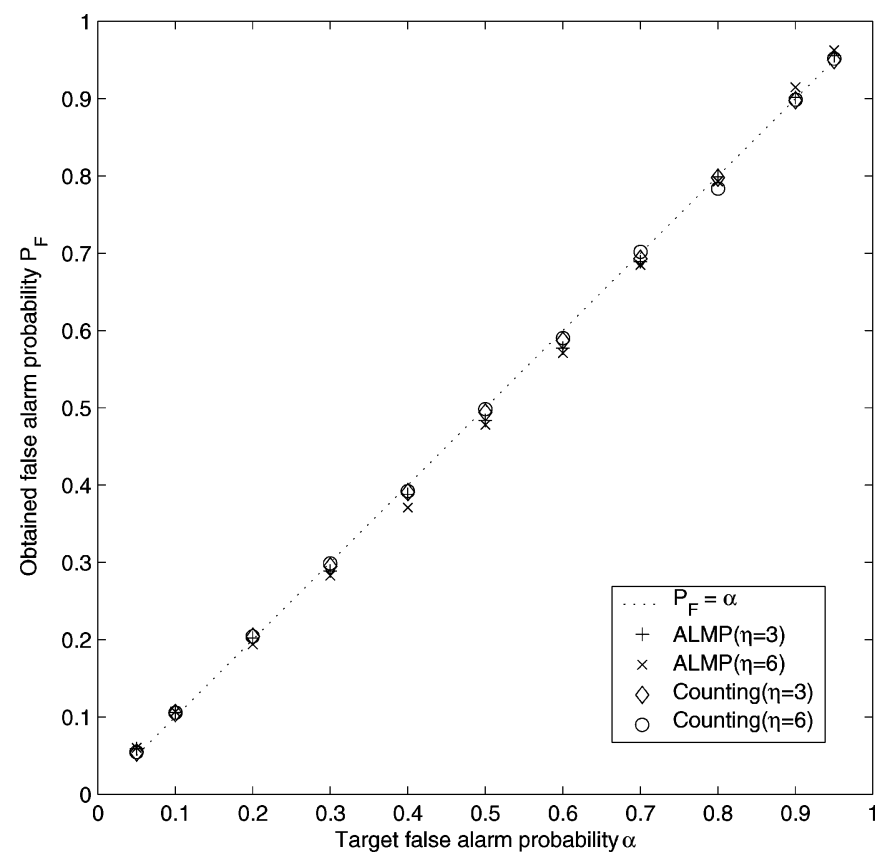

Fig. 6. Actual PFA versus the designed size.

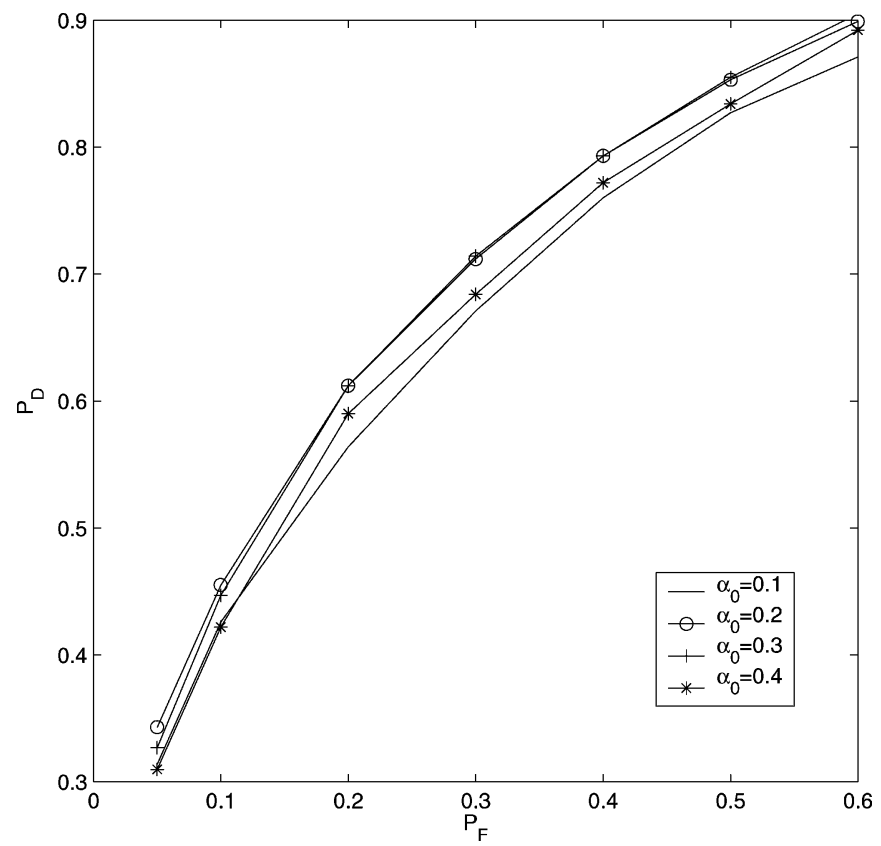

Fig. 7. ROC with different local sizes $(\theta=0.5)$.

the deviations are almost the same for the counting-based detector. This is because the test statistic $\Delta_{n, 0}$ for the ALMP detector is the sum of local decisions weighted by the spatial signal shape while the weight is uniform over the space for the counting-based detector. For the exponential with a large decay rate in (25), the sensor decisions around the origin dominate the overall distribution. Hence, the distribution is concentrated around the mean and deviates more from the Gaussian. This effect is more severe with a large decay rate $\eta=6$.

Fig. 7 shows the ROC of the ALMP detector using different local thresholds for a single sensor. The additive Gaussian input model was used and the average number of sensors in $A$ was

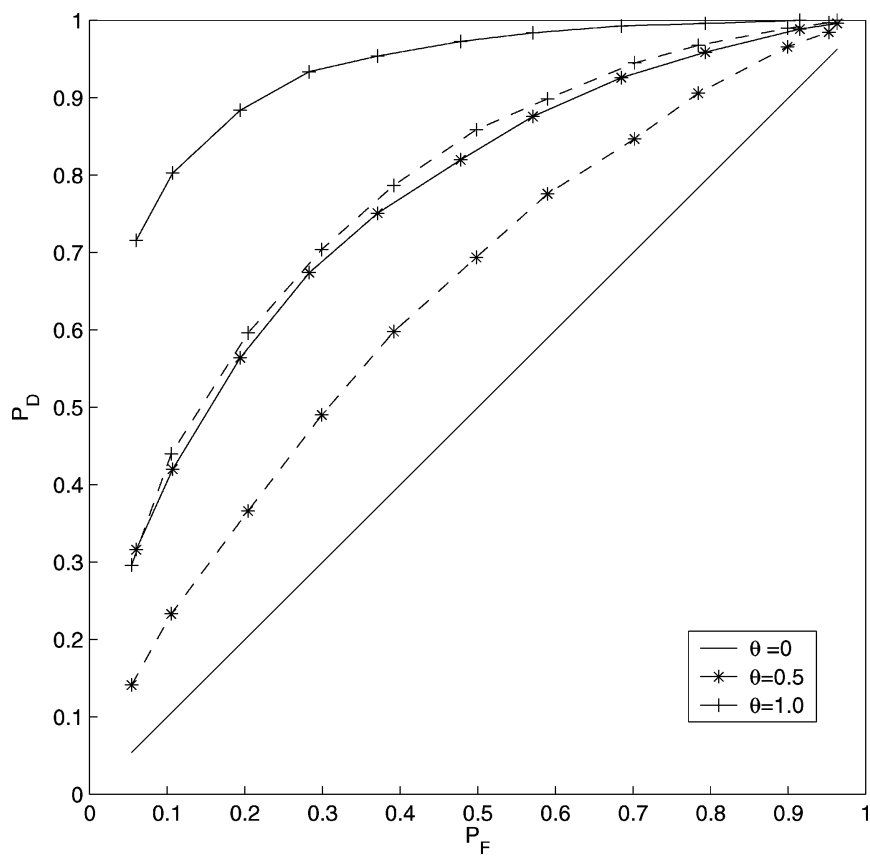

Fig. 8. ROC-Additive Gaussian sensor model. Solid line-ALMP detector. Dashed line-counting-based detector $(\eta=6)$.

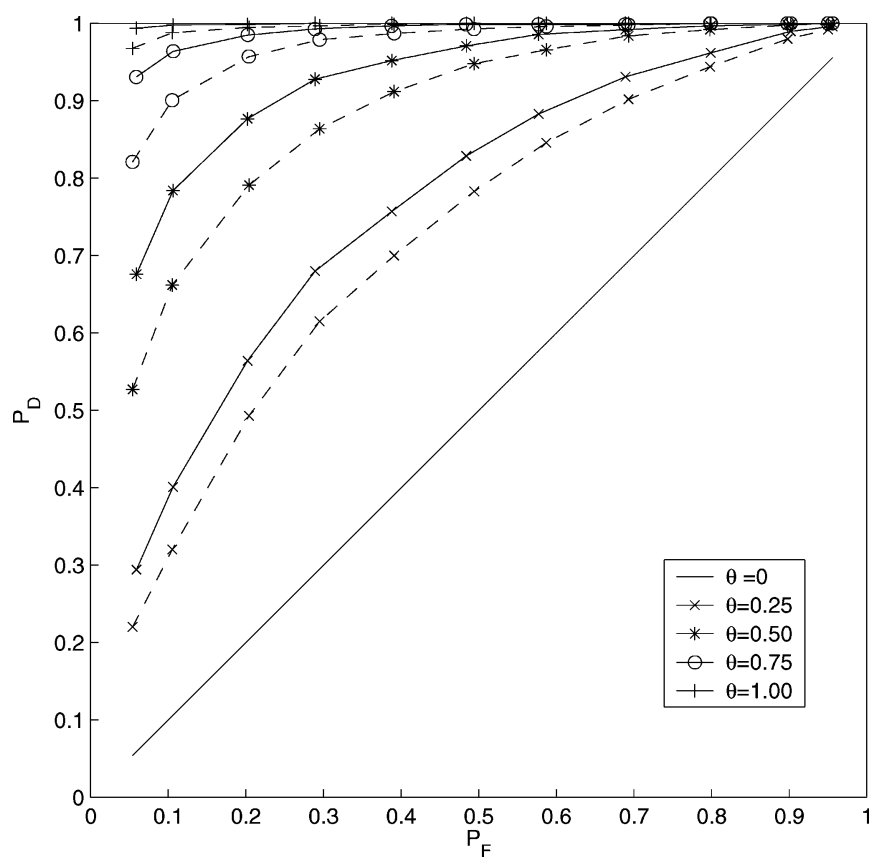

Fig. 9. ROC-Additive Gaussian sensor model. Solid line-ALMP detector. Dashed line-counting-based detector $(\eta=3)$.

kept the same at 1000 for all four cases in the figure. As shown, the global power changes with the local size of each sensor and the maximum is achieved between the local sizes of 0.2 and 0.3 , as predicted in Section III-B

Figs. 8 and 9 show the ROCs of the proposed ALMP and counting-based detector for the additive Gaussian sensor model with local size 0.1. Fig. 8 shows the case where the spatial signal changes quickly within the region $A$. The ALMP detector exploits spatial information and performs significantly better than the counting-based approach. Fig. 9 shows the ROCs for 
a smaller decay rate. As the decay rate becomes small or the signal becomes more uniform over the space, the performance gain by utilizing the spatial information becomes less significant. The meaning of asymptotic local optimality is evident in Fig. 9. In this case, we have a larger overall power in the space than the cases in Fig. 8 since the signal decays slowly over the space with the same peak at the origin. Since the peak value of $s(\mathbf{x})$ in $(25)$ is one and the variance for sensor input noise is chosen to be one, the maximum signal-to-noise ratio (SNR) for a sensor located at the origin is $0 \mathrm{~dB}$ when the amplitude parameter $\theta$ is one in the figure. Even though the SNR of $0 \mathrm{~dB}$ is very small for a single sensor, we have a large number of observations for the entire sensor field. (The average number is 1000 sensors.) Hence, the global power for $\theta=1$ already reaches almost unity for both the ALMP and counting-based detector, and the comparison above $\theta=1$ is less meaningful in this case. However, the performance within the local neighborhood of the null hypothesis is clearly distinguishable in all the figures.

Up to now, the true signal shape was used to obtain the ROC of ALMP detectors. Fig. 10(a) shows the ROC of the proposed detector with mismatched signal shapes. The true signal shape of POI was the symmetric exponential with $\eta=6$. We used two mismatched shapes as the weighting function to construct the central sequence. First, we considered the symmetric exponential $s_{1}(x, y)$ with a different decay rate $\eta=9$. As expected, the proposed detector with the mismatched shape performs worse than the true ALMP detector. However, for the case of $s_{1}(x, y)$ the performance almost approaches the ALMP detector since $s_{1}(x, y)$ is quite similar to the original shape. Therefore, we further approximated the signal shape by a step function

$$
s_{2}(x, y)= \begin{cases}1, & \sqrt{x^{2}+y^{2}} \leq r_{0} \\ 0, & \text { otherwise }\end{cases}
$$

where $r_{0}$ was determined such that the spatial "power" $s_{2}(x, y)$ covers $90 \%$ of that of the original signal, i.e., $\int_{A} s_{2}^{2}(x, y) d x d y=0.9 \int_{A} s^{2}(x, y) d x d y$. In this case, even though the degradation from the true ALMP becomes larger, it still shows good performance compared with the true ALMP detector. It is seen that rough knowledge of the signal shape is enough to get most of the advantage of the ALMP detector. Fig. 10(b) shows the ROC of the ALMP detector using the same signal shape but with mismatched centers. The same parameters were used as Fig. 10(a) for the true signal. The displacements of the center correspond to the positions of $80 \%, 60 \%$, and $40 \%$ from the amplitude of the true center. As shown, even with a rough estimate of the center, performance degradation is not severe compared with the perfectly matched case. Finally, the effect of inaccurate sensor locations was investigated. Fig. 10(c) shows the performance with the perturbed sensor locations. The same signal shape with $\eta=6$ was used for all ALMP detectors, but the perturbed locations of the sensors were used at the fusion center, i.e., $\hat{s}(\mathbf{x})=s(\mathbf{x}+\Delta \mathbf{x})$, where the perturbation $\Delta \mathbf{x}$ was generated independently for each sensor and was Gaussian distributed with standard deviations $1 \%, 5 \%$, and $10 \%$ of the radius of the total space. As shown by the figure, the ALMP detector is robust with respect to the sensor location errors and a rough estimate of the sensor location is enough.

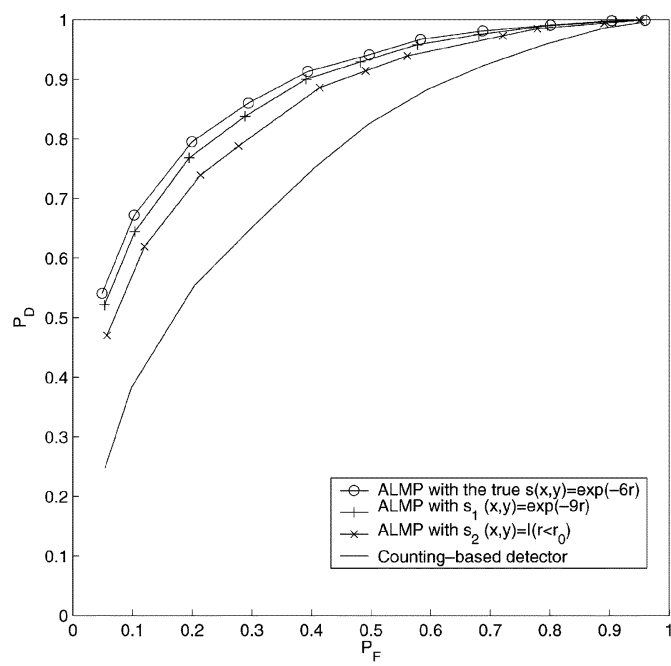

(a)

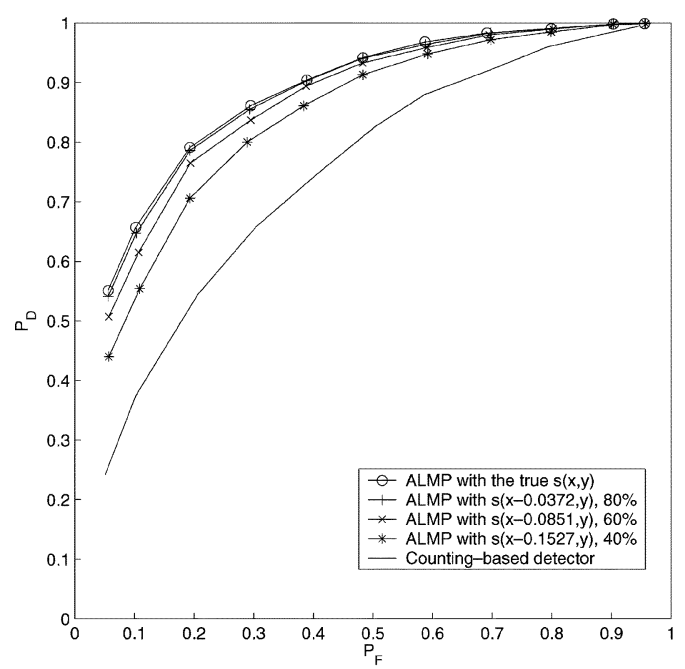

(b)

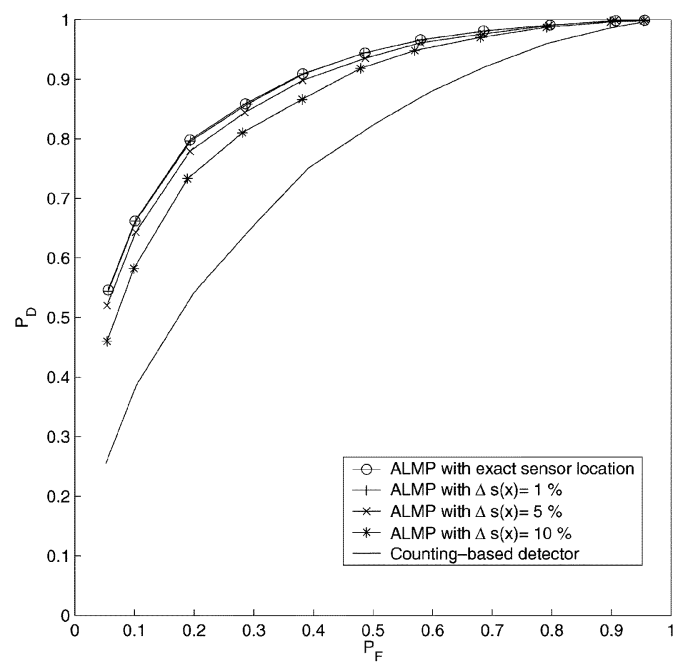

(c)

Fig. 10. ROC with mismatched signal shapes. (a) Mismatched rate. (b) Mismatched center. (c) Perturbed sensor location $\left(\alpha_{0}=0.27, \theta=0.75\right)$.

\section{CONCLUSION}

We considered a global detection problem based on (inaccurate) binary decisions from local threshold sensors. The 
local "alarm" probability is described by a generic function $\beta_{\tau_{0}}(\theta s(\mathbf{x}))$, where $\tau_{0}$ is the local threshold, $s(\mathbf{x})$ is the known underlying signal shape, and $\theta$ is the unknown amplitude. By assuming a homogeneous Poisson distribution of the sensors, we mapped the global detection problem to one of detecting Poisson processes with different intensities. Under a small signal strength assumption, asymptotically (in the number of sensors in a fixed area) locally most powerful (ALMP) detector was derived using LAN. It was shown that the conditions for applying LAN lead to reasonable restrictions on the underlying spatial signal. The ALMP fusion rule is a threshold detector for the weighted sum of local decisions, where the weight is proportional to the signal strength. The ALMP detector requires knowledge of the sensor locations, the signal shape, and a parameter which is the product of the local detector function $\beta_{\tau_{0}}(0)$, the MAC success probability, and the average density of the sensor locations. We have also shown (Theorem 3) how to optimize the local threshold to maximize the global power function. The asymptotic local optimality of the counting-based detector was established for the case of constant spatial signals. Numerical examples were provided to verify the theoretical results. Several of these examples also demonstrate that the proposed detector is robust under conditions of signal mismatch, including wrong signal shape, translated signal shape, and location calibration errors. Efficient estimators of $s(\mathbf{x})$, or its parameters, based on the binary sensor decisions, is currently under investigation.

\section{APPENDIX I}

\section{LOCAL ASYMPTOTIC NORMALITY (LAN) FOR THE MODEL IN SECTION II}

\section{A. Review of Poisson Processes}

The Poisson process $X_{A}$ in a metric space $A$ with a $\sigma$-field $\mathcal{B}$ is expressed in a simple manner by a counting measure notation as [9]

$$
X_{A}(B)=\sum_{i: \mathbf{x}_{i} \in A} \epsilon_{\mathbf{x}_{i}}(B), \forall B \in \mathcal{B}
$$

where $\mathbf{x}_{i}$ 's are random points in $A$ and

$$
\epsilon_{\mathbf{x}_{i}}(B) \triangleq \begin{cases}1, & \mathbf{x}_{i} \in B \\ 0, & \mathbf{x}_{i} \notin B\end{cases}
$$

The Poisson process has the following properties.

i) For every $B \in \mathcal{B}, X_{A}(B)$ is a Poisson random variable with mean $\Lambda(B)<\infty$.

ii) For every finite collection of disjoint sets $B_{1}, \ldots, B_{k} \in \mathcal{B}$, the random variables $X_{A}\left(B_{1}\right), \ldots$, $X_{A}\left(B_{k}\right)$ are independent.

Here, $\Lambda(\cdot)$ is called the intensity measure and its density ${ }^{4} \lambda(\mathbf{x})$, i.e., $\Lambda(d \mathbf{x})=\lambda(\mathbf{x}) d \mathbf{x}$, is called the (local) intensity. We define the stochastic integral for a given function $g$ as [7]

$$
I(g) \triangleq \int_{A} g(\mathbf{x}) X_{A}(d x)=\sum_{i: \mathbf{x}_{i} \in A} g\left(\mathbf{x}_{i}\right) .
$$

\footnotetext{
${ }^{4}$ For convenience, we assume that $\Lambda(\cdot)$ is differentiable.
}

The probability distribution of the Poisson process $X_{A}$ is determined by the local intensity. For the case of a parametric family of intensities such as (10), the probability distribution is also parameterized by the same parameter and given by [10]

$$
\begin{aligned}
d P_{\theta}\left(X_{A}\right) & =\exp \left(\int_{A} \log \lambda(\theta, \mathbf{x}) X_{A}(d \mathbf{x})-\int_{A} \lambda(\theta, \mathbf{x}) d \mathbf{x}\right) \\
& =\prod_{i: \mathbf{x}_{i} \in A} \lambda\left(\theta, \mathbf{x}_{i}\right) \exp \left(-\int_{A} \lambda(\theta, \mathbf{x}) d \mathbf{x}\right)
\end{aligned}
$$

The likelihood ratio between two distributions $P_{\theta_{0}}$ and $P_{\theta_{1}}$ is given by [7]

$$
\begin{aligned}
& \frac{d P_{\theta_{1}}}{d P_{\theta_{0}}}\left(X_{A}\right) \\
& =\exp \left(\int_{A} \log \frac{\lambda\left(\theta_{1}, \mathbf{x}\right)}{\lambda\left(\theta_{0}, \mathbf{x}\right)} X_{A}(d \mathbf{x})-\int_{A}\left[\lambda\left(\theta_{1}, \mathbf{x}\right)-\lambda\left(\theta_{0}, \mathbf{x}\right)\right] d \mathbf{x}\right) .
\end{aligned}
$$

\section{B. Construction of a Sequence of Statistical Models}

We construct a sequence of statistical models $\left(\Omega^{(n)}, \mathcal{X}^{(n)}, \mathcal{P}^{(n)}\right)$, where $\left(\Omega^{(n)}, \mathcal{X}^{(n)}\right)$ is the measurable space of all possible realizations of the Poisson process $X_{A_{n}}^{(n)}$ of the alarmed sensors on space $A_{n}$, and $\mathcal{P}^{(n)}$ is the corresponding family of probability distributions. Let $\lambda^{(n)}(\theta, \mathbf{x})$ be the local intensity of the Poisson process $X_{A_{n}}^{(n)}$. Then, the family of probability distributions $\mathcal{P}^{(n)}=\left\{P_{\theta}^{(n)}\left(X_{A_{n}}^{(n)}\right), \theta \in \Theta\right\}$ is given by (30). We are interested in the asymptotic scenario where the number of sensors deployed over a finite area goes to infinity. The model of increasing sensors in a finite area is described by increasing the initial intensity $\lambda_{h}$ of sensor deployment.

Model 1 (Finite Area and Infinite Sensor Model): We distribute sensors over space $A_{n}$ according to a homogeneous Poisson process with intensity $\lambda_{h}^{(n)}$ independently for each $n \geq 1$. We set

$$
A_{n}=A, \text { such that }|A|<\infty, \quad \text { for all } n=1,2, \ldots
$$

and choose the local intensity of initial sensor distribution over the space $A$ as

$$
\lambda_{h}^{(n)}=n \lambda_{h 0}
$$

Then, we collect the local decision of each sensor and observe the realization $X_{A}^{(n)}$ of the alarmed sensor distribution. For each $n \geq 1$, the local intensity of $X_{A}^{(n)}$ is given, using (10) and (11), by

$$
\lambda^{(n)}(\theta, \mathbf{x})=\theta n f(\mathbf{x})+n \lambda_{0}, \quad \theta \in \Theta
$$

where

$$
f(\mathbf{x})=\lambda_{h 0} p_{m} \beta_{\tau_{0}}^{\prime}(0) s(\mathbf{x}), \quad \lambda_{0}=\lambda_{h 0} p_{m} \beta_{\tau_{0}}(0)
$$

and the sequence of probability distributions $\left\{P_{\theta}^{(n)}, \theta \in \Theta\right\}$ is given, using (30), by

$$
\begin{aligned}
& d P_{\theta}^{(n)}\left(X_{A}^{(n)}\right) \\
& =\exp \left(\int_{A} \log \lambda^{(n)}(\theta, \mathbf{x}) X_{A}^{(n)}(d \mathbf{x})-\int_{A} \lambda^{(n)}(\theta, \mathbf{x}) d \mathbf{x}\right) .
\end{aligned}
$$


The LAN property of distributions of Poisson processes has been investigated by several authors [6]-[8] who derived the conditions in terms of the local intensity for LAN. However, the authors considered a sequence of models where the observation area $A_{n}\left(\subset A_{n+1}\right)$ goes to infinity for a fixed local intensity for all $n$, which is different from Model 1 . We derive new conditions in terms of the spatial signal shape for the LAN property of the model with increasing sensors in a finite area.

Theorem 3: For Model 1, suppose that $f(\mathbf{x})$ satisfies the following conditions

C.1) $f(\mathbf{x}) \geq 0, \mathbf{x} \in A$;

C.2) $\sup _{\mathbf{x} \in A} f(\mathbf{x})<\infty$;

C.3) $\int_{A} f(\mathbf{x}) d \mathbf{x}>0$.

Then, the statistical model $\left\{P_{\theta}^{(n)}, \theta \in \Theta\right\}$ of the alarmed sensor distribution $X_{A}^{(n)}$ is LAN at $\theta=0$, i.e., for every $h \geq 0$

$$
\log \frac{d P_{r_{n}(0) h}^{(n)}}{d P_{0}^{(n)}}\left(X_{A}^{(n)}\right)=h \Delta_{n, 0}-\frac{1}{2} h^{2}+o_{P_{0}^{(n)}}(1)
$$

where the central sequence $\Delta_{n, 0}$ and normalizing sequence $r_{n}(0)$ are given by

$$
\begin{aligned}
\Delta_{n, 0} & =\int_{A_{n}} r_{n}(0)\left(\frac{\dot{\lambda}^{(n)}(0, \mathbf{x})}{\lambda^{(n)}(0, \mathbf{x})}\right)\left[X_{A}^{(n)}(d \mathbf{x})-\Lambda_{0}^{(n)}(d \mathbf{x})\right] \\
r_{n}(0) & =J_{n}(0)^{-1 / 2}, J_{n}(0)=\int_{A}\left(\frac{\dot{\lambda}^{(n)}(0, \mathbf{x})}{\lambda^{(n)}(0, \mathbf{x})}\right)^{2} \Lambda_{0}^{(n)}(d \mathbf{x}) \\
\dot{\lambda}^{(n)}(\theta, \mathbf{x}) & =\frac{\partial}{\partial \theta} \lambda^{(n)}(\theta, \mathbf{x}), \Lambda_{0}^{(n)}(d \mathbf{x})=\lambda^{(n)}(0, \mathbf{x}) d \mathbf{x}=n \lambda_{h 0} d \mathbf{x}
\end{aligned}
$$

and $\mathcal{L}\left(\Delta_{n, 0} \mid P_{0}^{(n)}\right) \Rightarrow \mathcal{N}(0,1)$.

Proof: See Appendix II.

Here, the integration with random point measure in (38) is defined in (29). Condition C.1) requires that the single sensor power function $\beta_{\tau_{0}}(\cdot)$ must be a nondecreasing function at the origin $\theta=0$ for a given $\tau_{0}$ and that $s(\mathbf{x})$ be non-negative; C.2) is satisfied by any bounded $s(\mathbf{x})$, and $C .3$ ) says that $s(\mathbf{x})$ is not identically zero over the sensor field. The conditions C.1)-C.3) are general enough to include most interesting cases. Examples of allowed 2-D signal shapes for any region with a finite area include constant $f(\mathbf{x})$ or $s(\mathbf{x})$, a step function, Gaussian, or exponentially decaying signal (indeed any bounded non-negative function).

\section{APPENDIX II \\ PROOFS}

Lemma 1: Let $X_{A_{n}}^{(n)}$ be the sequence of Poisson processes (or corresponding statistical models) with probability distribution $\left\{P_{\theta}^{(n)}, \theta \in \Theta=[0, \infty)\right\}$ induced by the intensity measure $\Lambda_{\theta}^{(n)}(d \mathbf{x}) \triangleq \lambda^{(n)}(\theta, \mathbf{x}) d \mathbf{x}, \mathbf{x} \in A_{n}$. Let the conditions $B .1)-B .3)$ below be satisfied. Then, the statistical model $\left\{P_{\theta}^{(n)}, \theta \in \Theta\right\}$ is LAN at $\theta_{0} \in \Theta$ with central sequence $\Delta_{n}, \theta_{0}$ and normalizing sequence $r_{n}\left(\theta_{0}\right)$ defined in (38) and (39), respectively.

B.1) All intensity measures $\left\{\Lambda_{\theta}^{(n)}(d \mathbf{x}), \theta \in \Theta\right\}$, $n=1,2, \ldots$ are equivalent or mutually absolutely continuous for all $\theta \in \Theta$ and $\Lambda_{\theta}^{(n)}\left(A_{n}\right)<\infty$ for all $n$. We define

$$
S_{n}\left(\theta, \theta_{0} ; \mathbf{x}\right) \triangleq \frac{\Lambda_{\theta}^{(n)}(d \mathbf{x})}{\Lambda_{\theta_{0}}^{(n)}(d \mathbf{x})}=\frac{\lambda^{(n)}(\theta, \mathbf{x})}{\lambda^{(n)}\left(\theta_{0}, \mathbf{x}\right)}
$$

B.2) The function $S_{n}\left(\theta, \theta_{0} ; \mathbf{x}\right), \theta, \theta_{0} \in \Theta, x \in A_{n}$ is continuously differentiable with respect to $\theta$ at $\theta_{0}$. We define

$\Psi_{n}\left(\theta, \theta_{0} ; \mathbf{x}\right) \triangleq 2 \sqrt{S_{n}\left(\theta, \theta_{0} ; \mathbf{x}\right)}, \dot{\Psi}_{n}\left(\theta, \theta_{0} ; \mathbf{x}\right) \triangleq \frac{\partial}{\partial \theta} \Psi_{n}\left(\theta, \theta_{0} ; \mathbf{x}\right)$ $\dot{\Psi}_{n}\left(\theta_{0}, \theta_{0} ; x\right)=\frac{\dot{\lambda}^{(n)}\left(\theta_{0}, \mathbf{x}\right)}{\lambda^{(n)}\left(\theta_{0}, \mathbf{x}\right)}$.

Then, the quantity

$$
J_{n}\left(\theta_{0}\right) \triangleq \int_{A_{n}}\left|\dot{\Psi}_{n}\left(\theta_{0}, \theta_{0} ; \mathbf{x}\right)\right|^{2} \Lambda_{\theta_{0}}^{(n)}(d \mathbf{x})
$$

is positive $(>0)$ at $\theta_{0} \in \Theta$ and

$$
r_{n}\left(\theta_{0}\right) \triangleq J_{n}\left(\theta_{0}\right)^{-1 / 2} \rightarrow 0 \text { as } n \rightarrow \infty \text {. }
$$

B.3)

$$
\lim _{n \rightarrow \infty} \int_{A_{n}}\left|r_{n}\left(\theta_{0}\right) \dot{\Psi}_{n}\left(\theta_{0}, \theta_{0} ; \mathbf{x}\right)\right|^{3} \Lambda_{\theta_{0}}^{(n)}(d \mathbf{x}) \rightarrow 0
$$

and for every $C>0$, we have (43), shown at the bottom of the page.

Proof: The proof can be found in [6], [7], and [31].

Proof of Theorem 3: Since

$$
\lambda^{(n)}(\theta, \mathbf{x})=\theta n f(x)+n \lambda_{0}, \mathbf{x} \in A, \lambda_{0}>0
$$

for Model 1 and $f(\mathbf{x}) \geq 0, \mathbf{x} \in A$ by the condition $C .1)$, the family of intensity measures $\left\{\Lambda_{\theta}^{(n)}(d \mathbf{x})\right\}$ are equivalent for $\theta \geq$ 0 and all $n$. Hence, B.1) is satisfied, and $\Psi_{n}\left(\theta, \theta_{0} ; \mathbf{x}\right)$ is well defined, and its derivative is given by

$$
\dot{\Psi}_{n}\left(\theta, \theta_{0} ; \mathbf{x}\right)=\frac{\frac{n f(\mathbf{x})}{\theta n f(\mathbf{x})+n \lambda_{0}}}{\sqrt{\frac{\theta n f(\mathbf{x})+n \lambda_{0}}{\theta_{0} n f(\mathbf{x})+n \lambda_{0}}}}
$$

and at $\theta_{0}=0$

$$
\begin{aligned}
\dot{\Psi}_{n}(0,0 ; \mathbf{x}) & =\lambda_{0}^{-1} f(\mathbf{x}) \\
J_{n}(0) & =\int_{A_{n}}\left|\dot{\Psi}_{n}(0,0 ; \mathbf{x})\right|^{2} \Lambda_{0}^{(n)}(d \mathbf{x})=\int_{A} \lambda_{0}^{-2} f^{2}(\mathbf{x}) n \lambda_{0} d \mathbf{x} \\
& =n \lambda_{0}^{-1} \int_{A} f^{2}(\mathbf{x}) d \mathbf{x}
\end{aligned}
$$

$$
\lim _{n \rightarrow \infty} r_{n}^{2}\left(\theta_{0}\right) \sup _{|\theta-y|+|\theta-z|<r_{n}\left(\theta_{0}\right) C} \int_{A_{n}}\left[\frac{\dot{\lambda}^{(n)}(z, \mathbf{x})}{\lambda^{(n)}(y, \mathbf{x})}-\frac{\dot{\lambda}^{(n)}\left(\theta_{0}, \mathbf{x}\right)}{\lambda^{(n)}\left(\theta_{0}, \mathbf{x}\right)}\right]^{2} \Lambda_{\theta_{0}}^{(n)}(d \mathbf{x})=0 .
$$


since

$$
A_{n}=A \text { for all } n,|A|<\infty \text {. }
$$

By C.2) and C.3)

$$
0<C_{0}:=\int_{A} f^{2}(\mathbf{x}) d \mathbf{x}<\infty \Rightarrow 0<J_{n}(0)<\infty \text { for all } n
$$

and $\lim _{n \rightarrow \infty} J_{n}(0)=\infty$. Hence, B.2) is satisfied.

Define

$$
\begin{aligned}
r_{n}(0) & \triangleq J_{n}(0)^{-1 / 2}=n^{-1 / 2} \lambda_{0}^{1 / 2}\left[\int_{A} f^{2}(\mathbf{x}) d \mathbf{x}\right]^{-1 / 2} \\
& =n^{-1 / 2} \lambda_{0}^{1 / 2} C_{0}^{-1 / 2}
\end{aligned}
$$

Now, check (42).

$$
\begin{aligned}
\int_{A_{n}}\left|r_{n}(0) \dot{\Psi}_{n}(0,0 ; \mathbf{x})\right|^{3} \Lambda_{0}^{(n)}(d \mathbf{x}) \\
=\int_{A} n^{-3 / 2} \lambda_{0}^{3 / 2}\left[\int_{A} f^{2}(\mathbf{x}) d \mathbf{x}\right]^{-3 / 2} \lambda_{0}^{-3} f^{3}(\mathbf{x}) n \lambda_{0} d \mathbf{x} \\
=n^{-1 / 2} \lambda_{0}^{-1 / 2}\left[\int_{A} f^{2}(\mathbf{x}) d \mathbf{x}\right]^{-3 / 2} \int_{A} f^{3}(\mathbf{x}) d \mathbf{x} .
\end{aligned}
$$

Since $f(\mathbf{x}) \geq 0, \int_{A} f(\mathbf{x})>0$, and $\sup _{\mathbf{x} \in A} f(\mathbf{x})=M<\infty$

$$
0<\int_{A} f^{3}(\mathbf{x}) d \mathbf{x}<\infty .
$$

We have

$$
\int_{A_{n}}\left|r_{n}(0) \dot{\Psi}_{n}(0,0 ; \mathbf{x})\right|^{3} \Lambda_{0}(d \mathbf{x}) \rightarrow 0 \text { as } n \rightarrow \infty .
$$

Hence, the Lindeberg condition (42) is satisfied.

For given $C>0$

$$
\begin{aligned}
& \sup _{|y|+|z|<r_{n}(0) C} \int_{A_{n}} r_{n}^{2}(0)\left[\frac{\dot{\lambda}^{(n)}(z, \mathbf{x})}{\lambda^{(n)}(y, \mathbf{x})}-\frac{\dot{\lambda}^{(n)}(0, \mathbf{x})}{\lambda^{(n)}(0, \mathbf{x})}\right]^{2} \Lambda_{0}^{(n)}(d \mathbf{x}) \\
& =\sup _{0 \leq y<n^{-1 / 2} C^{\prime}} \int_{A} n^{-1} \lambda_{0}^{2} C_{0}^{-1}\left[\frac{f(x)}{y f(x)+\lambda_{0}}-\frac{f(x)}{\lambda_{0}}\right]^{2} n \lambda_{0} d x \\
& =\sup _{0 \leq y<n^{-1 / 2} C^{\prime}} \lambda_{0}^{3} C_{0}^{-1} \int_{A}\left[\frac{f(x)}{y f(x)+\lambda_{0}}-\frac{f(x)}{\lambda_{0}}\right]^{2} d x \\
& =\sup _{0 \leq y<n^{-1 / 2} C^{\prime}} \lambda_{0}^{3} C_{0}^{-1} \int_{A}\left[\frac{f(x)}{\lambda_{0}}\right]^{2}\left[\frac{1}{\frac{y f(x)}{\lambda_{0}}+1}-1\right]^{2} d x \\
& \left.\leq \sup _{0 \leq y<n^{-1 / 2} C^{\prime}} \lambda_{0}^{3} C_{0}^{-1}\left[\frac{1}{\frac{y M}{\lambda_{0}}+1}-1\right]_{A}^{2} \frac{f(x)}{\lambda_{0}}\right]^{2} d x \\
& \leq \lambda_{0}^{3} C_{0}^{-1}\left[\frac{1}{\frac{n^{-1 / 2} C^{\prime} M}{\lambda_{0}}+1}-1\right]_{A}^{2}\left[\frac{f(x)}{\lambda_{0}}\right]^{2} d x \\
& \rightarrow 0 \text { as } n \rightarrow \infty .
\end{aligned}
$$

where $C^{\prime}=\lambda_{0}^{1 / 2} C_{0}^{-1 / 2} C$. Here, we used the fact that $h(x)$ defined in (46) is monotone increasing for $x \geq 0$.

$$
h(x)=\left(\frac{1}{a x+1}-1\right)^{2}, \text { for any } a>0 .
$$

Hence, B.3) is satisfied.

Proof of Theorem 1:

$$
\begin{aligned}
& \frac{\dot{\lambda}^{(n)}(0, \mathbf{x})}{\lambda^{(n)}(0, \mathbf{x})}=\left.\frac{n f(\mathbf{x})}{\theta n f(\mathbf{x})+n \lambda_{0}}\right|_{\theta=0}=\lambda_{0}^{-1} f(\mathbf{x}) \\
& r_{n}(0)=\left[\int_{A}\left(\frac{\dot{\lambda}^{(n)}(0, \mathbf{x})}{\lambda^{(n)}(0, \mathbf{x})}\right)^{2} \lambda^{(n)}(0, \mathbf{x}) d \mathbf{x}\right]^{-1 / 2} \\
& =n^{-1 / 2} \lambda_{0}^{1 / 2}\left(\int_{A} f^{2}(\mathbf{x}) d \mathbf{x}\right)^{-1 / 2} \\
& \Delta_{n, 0}=r_{n}(0) \int_{A} f(\mathbf{x})\left[X^{(n)}(d \mathbf{x})-\Lambda_{0}^{(n)}(d \mathbf{x})\right] \\
& =n^{-1 / 2} \lambda_{0}^{-1 / 2}\left(\int_{A} c^{2} s^{2}(\mathbf{x}) d \mathbf{x}\right)^{-1 / 2} \\
& \cdot\left(\int_{A} c s(\mathbf{x}) X^{(n)}(d \mathbf{x})-n \lambda_{0} \int_{A} c s(\mathbf{x}) d \mathbf{x}\right) \\
& =n^{-1 / 2} \lambda_{0}^{-1 / 2}\left(\int_{A} s^{2}(\mathbf{x}) d \mathbf{x}\right)^{-1 / 2} \\
& \cdot\left(\sum_{i: \mathbf{x}_{i} \in A} s\left(\mathbf{x}_{i}\right)-n \lambda_{0} \int_{A} s(\mathbf{x}) d \mathbf{x}\right) \text {. }
\end{aligned}
$$

Here, we used the fact that $f(\mathbf{x})$ is a scaled version of $s(\mathbf{x})$ $(f(\mathbf{x})=c s(\mathbf{x}), c>0)$. The last step is by the definition of stochastic integral. The ALMP detector is obtained by the score test [4].

Proof of Theorem 2: By Theorem 1, the asymptotic local upper bound for the global power is given by

$$
Q\left(Q^{-1}(\alpha)-r_{n}^{-1}(0) \theta\right) .
$$

Since $Q(\cdot)$ is a monotone decreasing function, the maximum upper bound for a fixed $\theta$ is achieved by maximizing $r_{n}^{-1}(0)$ for a given $n$. Since $r_{n}(0)$ is given, using (35), by

$$
\begin{aligned}
r_{n}(0) & =n^{-1 / 2} \lambda_{0}^{1 / 2}\left[\int_{A} f^{2}(\mathbf{x}) d \mathbf{x}\right]^{-1 / 2} \\
& =\left(n \lambda_{h 0} p_{m} \frac{\left(\beta_{\tau_{0}}^{\prime}(0)\right)^{2}}{\beta_{\tau_{0}}(0)} \int_{A} s^{2}(\mathbf{x}) d \mathbf{x}\right)^{-1 / 2}
\end{aligned}
$$

the theorem follows.

\section{REFERENCES}

[1] L. Le Cam, "Locally asymptotically normal families of distributions," Univ. Calif. Pub. Statist., vol. 3, no. 2, pp. 37-98, 1960.

[2] L. Le Cam and G. L. Yang, Asymptotics in Statistics: Some Basic Concepts, Second ed. New York: Springer-Verlag, 2000.

[3] G. G. Roussas, Contiguity of Probability Measures: Some Applications in Statistics. Cambridge, U.K.: Cambridge Univ. Press, 1972.

[4] A. W. van der Vaart, Asymptotic Statistics. Cambridge, U.K.: Cambridge Univ. Press, 1998.

[5] A. N. Shiryaev, Probability, Second ed. New York: Springer-Verlag, 1995.

[6] Y. A. Kutoyants, "Locally asymptotic normality for processes of poisson type," Soviet J. Contemporary Math. Anal., vol. 14, pp. 1-18, 1979.

[7] - Statistical Inference for Spatial Poisson Processes. New York: Springer-Verlag, 1998.

[8] F. Liese and U. Lorz, "Contiguity and LAN-property of sequences of poisson processes," Kybernetika, vol. 35, no. 3, pp. 281-308, 1999.

[9] A. F. Karr, Point Processes and their Statistical Inference. New York: Marcel Dekker, 1986. 
[10] D. R. Snyder and M. I. Miller, Random Point Processes in Time and Space. New York: Springer-Verlag, 1991.

[11] I. Bar-David, "Communications under the poisson regime," IEEE Trans. Inf. Theory, vol. IT-15, no. 1, pp. 31-37, Jan. 1969.

[12] J. N. Tsitsiklis, "Decentralized detection," in Advances in Statistical Signal Processing, H.V. Poor and J.B. Thomas, Eds. Greenwich, CT: JAI, 1990, vol. 2, Signal Detection.

[13] R. Viswanathan and P. K. Varshney, "Distributed detection with multiple sensors: Part I-Fundamentals,” Proc. IEEE, vol. 85, no. 1, pp. 54-63, Jan. 1997.

[14] R. S. Blum, S. A. Kassam, and H. V. Poor, "Distributed detection with multiple sensors: Part II-Advanced topics," Proc. IEEE, vol. 85, no. 1, pp. 64-79, Jan. 1997.

[15] R. R. Tenney and N. R. Sandell, "Detection with distributed sensors," IEEE Trans. Aerosp. Electron. Syst., vol. AES-17, no. 4, pp. 501-509, Jul. 1981.

[16] Z. Chair and P. K. Varshney, "Optimal data fusion in multiple sensor detection systems," IEEE Trans. Aerosp. Electron. Syst., vol. 22, no. AES-1, pp. 98-101, Jan. 1986.

[17] S. C. A. Thomopoulos, R. Viswanathan, and D. K. Bougoulias, "Optimal distributed detection fusion," IEEE Trans. Aerosp. Electron. Syst., vol. 25, no. 5, pp. 761-765, Sep. 1989.

[18] Q. Yan and R. S. Blum, "Distributed signal detection under the Neyman-Pearson criterion," IEEE Trans. Inf. Theory, vol. 47, no. 4, pp. 1368-1377, May 2001.

[19] R. Viswanathan and V. Aalo, "On counting rules in distributed detection," IEEE Trans. Acoust., Speech, Signal Process., vol. 37, no. 5, pp. 772-775, May 1989.

[20] J. Capon, "On the asymptotic efficiency of locally optimum detectors," IRE Trans. Inf. Theory, vol. 7, pp. 67-71, Apr. 1961.

[21] H. V. Poor, An Introduction to Signal Detection and Estimation, Second ed. New York: Springer, 1994

[22] H. V. Poor and J. B. Thomas, "Locally optimum detection of discretetime signals in non-Gaussian noise," J. Acoust. Soc. Amer, vol. 63, no. 1, pp. 75-80, Jan. 1978.

[23] S. A. Kassam and J. B. Thomas, "Dead-zone limiter: An application of conditional tests in nonparametric detection," J. Acoust. Soc. Amer, vol. 60 , no. 4, pp. 857-862, Oct. 1976

[24] V. Aalo and R. Viswanathan, "Multilevel quantization and fusion scheme for the decentralized detection of an unknown signal," Proc. Inst. Elect. Eng. Radar, Sonar Navig., vol. 141, no. 1, pp. 37-44, Feb. 1994.

[25] G. Fedele, L. Izzo, and L. Paura, "Optimum and suboptimum spacediversity detection of weak signals in non-Gaussian noise," IEEE Trans. Commun., vol. COM-32, no. 9, pp. 990-997, Sep. 1984

[26] R. Srinivasan, "The detection of weak signals using distributed sensors," in Proc. Conf. Inf. Sci. Syst., Princeton, NJ, Mar. 1990, pp. 541-545.

[27] J. N. Tsitsiklis, "Decentralized detection by a large number of sensors," Math. Contr. Signals Syst., no. 1, pp. 167-182, 1988.

[28] P.-N. Chen and A. Papamarcou, "New asymptotic results in parallel distributed detection," IEEE Trans. Inf. Theory, vol. 39, no. 6, pp. 1847-1863, Nov. 1993.

[29] J.-F. Chamberland and V. Veeravalli, "Decentralized detection in sensor networks," IEEE Trans. Signal Processing, vol. 51, no. 2, pp. 407-416, Feb. 2003.

[30] L. Tong, Q. Zhao, and S. Adireddy, "Sensor networks with mobile agents," in Proc. MILCOM, Boston, MA, Oct. 2003.

[31] Y. Sung, L. Tong, and A. Swami. (2003) "Asymptotic Locally Optimal Detector for Large Scale Sensor Networks under the Poisson Regime," Tech. Rep. ACSP 10.03.01. Cornell Univ., Sch. Elect. Comput. Eng., Adaptive Commun. Signal Process. Lab.. [Online]. Available: http://acsp.ece.cornell.edu

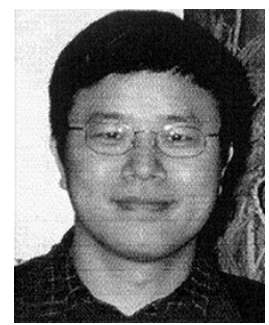

Youngchul Sung received the B.S. and M.S. degrees in electronic engineering from Seoul National University, Seoul, Korea, in electronics engineering in 1993 and 1995, respectively, and the Ph.D. degree from Cornell University, Ithaca, NY, in May 2005.

$\mathrm{He}$ was a research engineer at Goldstar Information and Communications, Ltd., Seoul, from 1995 to 2000. At Goldstar, he developed high data-rate wireless transmission systems and wireless local loop based on wideband code-division multiple-access (WCDMA). His areas of interest include statistical signal processing and communication networks, especially in signal processing techniques and asymptotic statistical methods for communication systems and large-scale sensor networks.

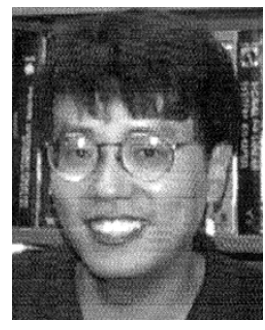

Lang Tong (S'87-M'91-SM'01-F'05) received the B.E. degree from Tsinghua University, Beijing, China, in 1985 and the M.S. and Ph.D. degrees in electrical engineering in 1987 and 1990, respectively, from the University of Notre Dame, Notre Dame, IN

He was a Postdoctoral Research Affiliate at the Information Systems Laboratory, Stanford University, Stanford, CA, in 1991. Currently, he is a Professor with the School of Electrical and Computer Engineering, Cornell University, Ithaca, NY. His areas of interest include statistical signal processing, adaptive receiver design for communication systems, signal processing for communication networks, and information theory.

Dr. Tong received Young Investigator Award from the Office of Naval Research in 1996 and the Outstanding Young Author Award from the IEEE Circuits and Systems Society. He also received (with M. Dong) the 2004 IEEE Signal Processing Society Best Paper Award.

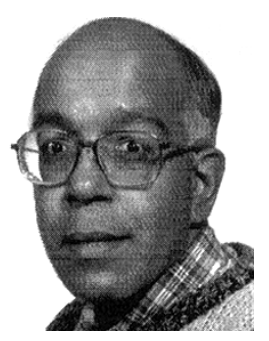

Ananthram Swami (SM'96) received the B.S degree from the Indian Institute of Technology, Bombay, India; the M.S. degree from Rice University, Houston, TX; and the Ph.D. degree from the University of Southern California, Los Angeles, all in electrical engineering.

He has held positions with Unocal Corporation, the University of Southern California, CS-3, and Malgudi Systems. He is currently a Senior Research Scientist with the U.S. Army Research Lab, Adelphi, $\mathrm{MD}$, where his work is in the broad area of signal processing for communications. He was a Statistical Consultant to the California Lottery, developed a Matlab-based toolbox for non-Gaussian signal processing, and has held visiting faculty positions at INP, Toulouse, France.

Dr. Swami is a member and vice-chair of the IEEE Signal Processing Society's Technical Committee on Signal Processing for Communications, an associate editor of the IEEE TRANSACTIONS ON WIRELESS COMMUNICATIONS, and of the IEEE TRANSACTIONS ON SIGNAL PROCESSING, and a member of the Editorial Board of the IEEE SIGNAL PROCESSING MAGAZINE. He has served as an associate editor for IEEE SIGNAL PROCESSING LETTERS and IEEE TRANSACTIONS ON CIRCUITS AND SYSTEMS II. He was co-organizer and co-chair of the 1993 IEEE-SPS HOS Workshop, the 1996 IEEE-SPS SSAP Workshop, and the 1999 ASA-IMA Workshop on Heavy-Tailed Phenomena. He is co-guest editor of a 2004 special issue of the IEEE SignAL PROCESSING MAGAZINE on "signal processing for networking." 